\title{
PRIMORDIAL MAGNETIC FIELDS BY COSMIC INFLATION
}

\author{
SHU-LIN CHENG and WO-LUNG LEE \\ Department of Physics, National Taiwan Normal University, \\ Taipei, Taiwan 116, Republic of China \\ leewl@phy.ntnu.edu.tw \\ KIN-WANG NG \\ Institute of Physics, Academia Sinica \\ Taipei, Taiwan 115, Republic of China \\ nkw@phys.sinica.edu.tw
}

\begin{abstract}
Contrary to the conventional wisdom, we find that it is plausible to generate primordial magnetic seed fields via spinodal instability efficiently during cosmic inflation provided that a fast-roll stage is involved before the inflaton entering into the slow-roll phase. Moreover, the primordial magnetic field produced in such a mechanism can be used to constrain the low quadrupole moment of Cosmic Microwave Background.
\end{abstract}

Keywords: Primordial magnetic field; inflation; spinodal Instability.

\section{Introduction}

Presently the origin still remains elusive for the observed galactic and extragalactic magnetic fields of about a few $\mu \mathrm{G}^{1,2}$. It is generally believed that the so-called galactic dynamo ${ }^{3}$ mechanism must be involved to amplify a magnetic seed field $B_{\text {seed }} \sim 10^{-23} \mathrm{G}$ on a comoving scale larger than Mpc. Since the Universe is in a highly conducting plasma state through the most of its history, the ratio of the magnetic energy density and the thermal background, $\rho_{B} / \rho_{\gamma}$, remains constant along the cosmic evolution. The required $B_{\text {seed }}$ then translates into $\rho_{B} \simeq 10^{-34} \rho_{\gamma}$.

Depending on the correlation scale of the magnetic field, the seed fields can be produced inside or outside the Hubble sphere. Various astrophysical hypotheses, e.g. the Biermann battery ${ }^{4}$, and cosmological scenarios, e.g. Harrison's vorticity model ${ }^{5}$, may generate large magnetic fields locally. Even if inverse cascades are invoked, however, the correlation scales of the resultant fields will not extend beyond 100 $\mathrm{pc}^{6}$. Apparently these processes are incapable of explaining the observed cosmic magnetic fields which are coherent over much larger scales.

On the other hand, the correlation scale of primordial seed fields produced during inflation in the early Universe is much greater than the Hubble radius. As we know, 
the de Sitter like expansion is better described by the spatially flat FriedmannRobertson-Walker (FRW) metric which conformally preserves the standard electromagnetic (EM) action ${ }^{7}$. Therefore, a viable inflationary magnetogenesis scenario must exploit some mechanism to break the conformal invariance of the EM field. There are several popular methods to serve the purpose. For examples, one can consider the EM field tensor $F$ coupling to the gravitational curvature scalar $R$ in the forms ${ }^{8}$ of $R A^{2}, R_{\mu \nu} A^{\mu} A^{\nu}, R F^{\mu \nu} F_{\mu \nu}$, where $F_{\mu \nu}=\partial_{\mu} A_{\nu}-\partial_{\nu} A_{\mu}$ and $A^{\mu}$ is the vector potential; to scalar inflatons or dilations ${ }^{8,9}$; to pseudo-scalar fields like axion ${ }^{10} ; \ldots$ etc. However, most of them do not produce satisfactory outcomes ${ }^{11,12,13,14}$.

Motivated by the mechanism of axial couplings ${ }^{10}$, we have developed a scenario connecting the primordial magnetic field (PMF) to the dark energy ${ }^{15}$. Assuming that an evolving pseudo scalar field is responsible for the late time acceleration of the Universe ${ }^{16}$, a sufficiently large PMF can be produced inside the Hubble sphere via the spinodal instability. Furthermore, the spinodal effect is capable of generating long-wavelength fluctuations where the created magnetic fields correspond to coherent collective behavior in which the fields correlate themselves over a large distance of order of $10 \mathrm{Mpc}$.

In this paper, we investigate the origin of PMF outside the Hubble radius by applying the same formalism of the EM coupling to a pseudo scalar inflaton $\phi$. Once again we find that the spinodal instability provides a robust mechanism for generating PMF during the inflation epoch. However, a pre-inflationary fast roll stage must be involved in order to increase the efficiency of PMF production. The correlation length of the resultant seed fields is around $100 \mathrm{Mpc}$.

This paper is organized as follows. In the next section, we present our formulation for the axial coupling of the EM field to the pseudo scalar inflaton. We employ the chaotic inflation scenario to carry out the numerical calculation and show that the created PMF is not sufficient in Sec. 3. We then re-analyze our solutions by taking into account a pre-inflationary fast-roll stage. Finally, we conclude with a few comments on our results.

\section{Axial coupling of the EM field to a pseudo scalar inflaton}

We consider a cosmic inflation characterized by the action

$$
S=\int d^{4} x \sqrt{g}\left\{\left[-\frac{1}{2} g^{\mu \nu} \partial_{\mu} \phi \partial_{\nu} \phi-V(\phi)\right]-\frac{1}{4} g^{\alpha \mu} g^{\beta \nu} F_{\alpha \beta} F_{\mu \nu}+\frac{1}{\sqrt{g}} L_{\phi \gamma}\right\},
$$

where the signature is $(-+++)$ and $L_{\phi \gamma}$ is the $\phi$-photon coupling given by

$$
L_{\phi \gamma}=\frac{c}{M_{\mathrm{Pl}}} \phi \epsilon^{\alpha \beta \mu \nu} F_{\alpha \beta} F_{\mu \nu}
$$

in which $M_{\mathrm{Pl}} \equiv 1 / \sqrt{G}$ denotes the Planck mass. In this study we treat the coupling constant $c$ as a free parameter. The background evolution is governed by the equation of motion

$$
\ddot{\varphi}+3 \mathcal{H} \dot{\varphi}+V^{\prime}(\varphi)=0,
$$


and the Hubble expansion characterized by

$$
\mathcal{H}^{2}=\left(\frac{H}{M_{\mathrm{Pl}}}\right)^{2}=\frac{8 \pi}{3}\left[\frac{\dot{\varphi}^{2}}{2}+V(\varphi)\right],
$$

where $\varphi \equiv \phi / M_{\mathrm{Pl}}$, and the dot represents differentiation with respect to the rescaled cosmic time $\tilde{t} \equiv M_{\mathrm{Pl}} \cdot t$.

In the slow roll regime where the Hubble radius $H^{-1}$ remains more or less a constant, the comoving magnetic field can be expressed in terms of the comoving coordinates $(\tau, \mathbf{x})$ with the re-scaled conformal time $\tau \equiv \eta / H$ as ${ }^{8,10,15}$

$$
\left(\nabla^{2}-\frac{\partial^{2}}{\partial \tau^{2}}\right) \mathbf{B}=4 c \frac{d \varphi}{d \tau} \nabla \times \mathbf{B},
$$

where $\nabla \equiv \partial / \partial \mathbf{x}$, and the conformal time $\eta$ satisfies $d \eta=d t / a$. The magnetic field can be casted as $\mathbf{B}=\nabla \times \mathbf{A}_{T}$. Moreover, the transverse field $\mathbf{A}_{T}(\tau, \mathbf{x})$ can be decomposed into Fourier modes such that

$$
\mathbf{A}_{T}=\int \frac{d^{3} \mathbf{k}}{\sqrt{2(2 \pi)^{3} k}}\left[e^{i \mathbf{k} \cdot \mathbf{x}} \sum_{\lambda= \pm} b_{\lambda \mathbf{k}} V_{\lambda \mathbf{k}}(\tau) \epsilon_{\lambda \mathbf{k}}+\text { h.c. }\right]
$$

where $b_{ \pm \mathbf{k}}$ are destruction operators, and $\epsilon_{ \pm \mathbf{k}}$ are circular polarization unit vectors. Then, defining $q=k / H$, the mode equations are obtained from Eqs. (5) and (6) as

$$
\frac{d^{2}}{d \eta^{2}} V_{ \pm q}+\left(q^{2} \mp 4 c q \frac{d \varphi}{d \eta}\right) V_{ \pm q}=0,
$$

with initial conditions at early epoch given by

$$
V_{ \pm q}=1, \quad \frac{d V_{ \pm q}}{d \eta}=-i q
$$

Hence, the comoving energy density of the magnetic field is given by $\rho_{B}=$ $\left\langle B^{2}\right\rangle / 8 \pi=\int d q\left(d \rho_{B} / d q\right)$ with

$$
\frac{d \rho_{B}}{d q}=\frac{q^{3}}{32 \pi^{3}}\left(\frac{H}{a_{\mathrm{end}}}\right)^{4} \sum_{\lambda= \pm}\left|V_{\lambda q}\right|^{2},
$$

where $a_{\mathrm{end}}$ is the scale factor at the end of the inflation epoch.

According to the mode equation (8), it is evident that the conformal invariance of the EM field will be broken only when the coupling constant $c \neq 0$. In fact, since

$$
V_{ \pm} \propto \exp \left[\left( \pm 4 c q\left|\frac{d \varphi}{d \eta}\right|-q^{2}\right)^{1 / 2} \eta\right]
$$

the fluctuation modes lying within the unstable band with $q<4 c|d \varphi / d \eta|$ will always get amplified exponentially. Therefore, the spinodal instability does provide a robust mechanism for producing PMF. 


\section{The slow-rolling is not enough}

Employing the chaotic inflation scenario ${ }^{17}$ in which the inflaton potential is given by

$$
V(\phi)=\frac{m^{2}}{2} \phi^{2}
$$

we solve for the ratio of the spectral magnetic energy density [Eq. (9)] to the energy density of photon $\rho_{\gamma}$ at the end of inflation. Assuming the reheating temperature in the post inflationary epoch is $H, \rho_{\gamma}$ can be derived from the energy density of the radiation component as

$$
\rho_{\gamma}=\frac{\pi^{2}}{15} g_{\mathrm{eff}} H^{4}
$$

where $g_{\text {eff }}=106.75$ is used. Since $\rho_{B} / \rho_{\gamma}$ is preserved in most of the cosmic history, the peak value of the ratio $r$ must satisfy the following condition for a viable magnetogenesis process while keeping the total e-folding growth $N=\int H d t=60$ at the end of inflation:

$$
r \equiv \rho_{\gamma}^{-1}\left[\frac{d \rho_{B}}{d q}\right]_{\max } \simeq 10^{-34} .
$$

Taking $m=1.8 \times 10^{13} \mathrm{GeV}$ for the inflaton mass, and $\varphi_{i}=3.0, \dot{\varphi}_{i}=-2.5 \times 10^{-7}$ respectively for the initial position and velocity of the inflaton $\varphi$, we find that the coupling constant must be as large as $c=20500$ to fit the requirement that $\log r=-34$, as described in Fig. 1. On the other hand, changing the mass scale $m$ of the inflaton while fixing a reasonable $c$ and all other initial conditions does not remedy the situation. Apparently, the megnetogenesis induced by the spinodal instability in the slow roll regime is not efficient enough. We are forced to consider invoking a fast roll stage prior to the slow roll phase.

Figure 2 shows a $\varphi-\dot{\varphi}$ phase plane for the chaotic inflation. The attractor solution indicated by the dashed line corresponds to the slow roll regime of inflation. Before entering the slow roll solution, there exists an ultra-hard region where $|\dot{\varphi}| \gg m \varphi^{18}$. Because the kinetic energy dominates in this region, it can be identified as a fast roll stage. When an inflaton being placed in such a fast roll regime, it will experience a rapid deceleration in order to entering into the normal slow roll track of inflation. In terms of the axial coupling mechanism, the ditched kinetic energy is converted by the spinodal instability into the magnetic energy. Hence, a boost in magetic power is anticipated in this situation.

As an example, we plot the evolutions of $\varphi$ and $\dot{\varphi}$ in Fig. 3 when initial values $\varphi_{i}=4.35$ and $\dot{\varphi}=-0.01$ are taken into account. In our calculations the number of e-folds is fixed at $N=60$. The change in $\dot{\varphi}$ is so rapid that the fast roll period only lasts for about $3 \%$ of the total number of e-folds. However, this kinetic effect has improved our scenario of magnetogenesis a lot.

The ratio of the spectral magnetic energy density to the energy density of photon at the end of inflation in this case is shown in Fig. 4. The coupling constant in this 


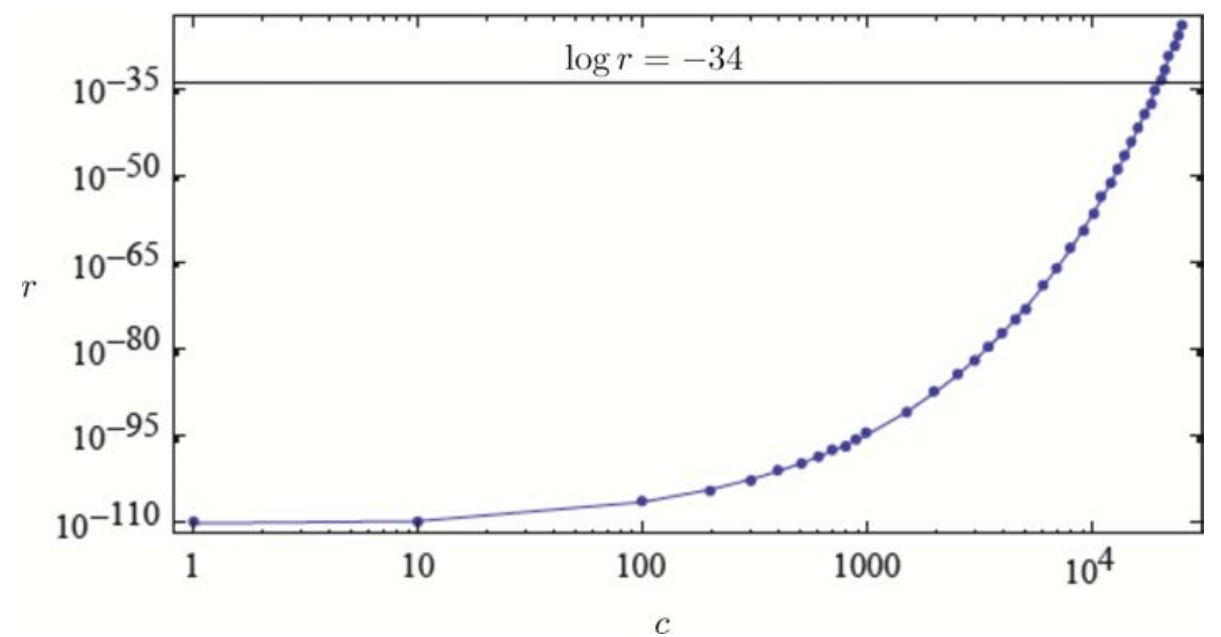

Fig. 1. The r vs c diagram suggests that the megnetogenesis induced by the spinodal instability in the slow roll regime is not efficient enough.

case is $c=34.5$. The efficiency of spinodal instability is about 6000 times better than that in the situation when only a slow roll phase is taken into account. This is one of the great merit of the pre-inflationary fast roll stage.

\section{Conclusions}

We have studied the inflationary magnetogenesis by considering an axial coupling between the EM field and a pseudo scalar inflaton. The resultant PMF is helical revealing the violation of the parity symmetry. Because that the conservation of helicity protects B fields from any possible destruction through the evolution of the early universe, the spinodal instability indeed provides a robust mechanism to create magnetic seed fields. However, as many authors have shown, the efficiency of magnetic field production is quite low when the generation process involves only the slow roll regime of inflation. If one takes also a pre-inflationary fast roll stage into account, the kinetic effect enhances the magnetogenesis significantly.

As we know, there is a stringent limit on the axial coupling of EM field to a light pseudo scalar $\phi^{19}$. However, there exists no such a constraint for the coupling to a massive pseudo scalar field. Due to the stiffness nature of the ultra-hard fast roll regime, we are incapable of analyzing the magnetogenesis beyond $\dot{\varphi} \simeq-0.001$ without resorting to further approximation. If we require the theory to be perturbative, a new energy scale may need to be introduced into the scenario.

Moreover, the enhancement in PMF production by the pre-inflationary fast roll phase will inevitably suppress large-scale inflaton fluctuations. Such a process will give rise to a lower large-scale CMB anisotropy. This is worth of further investigation. 


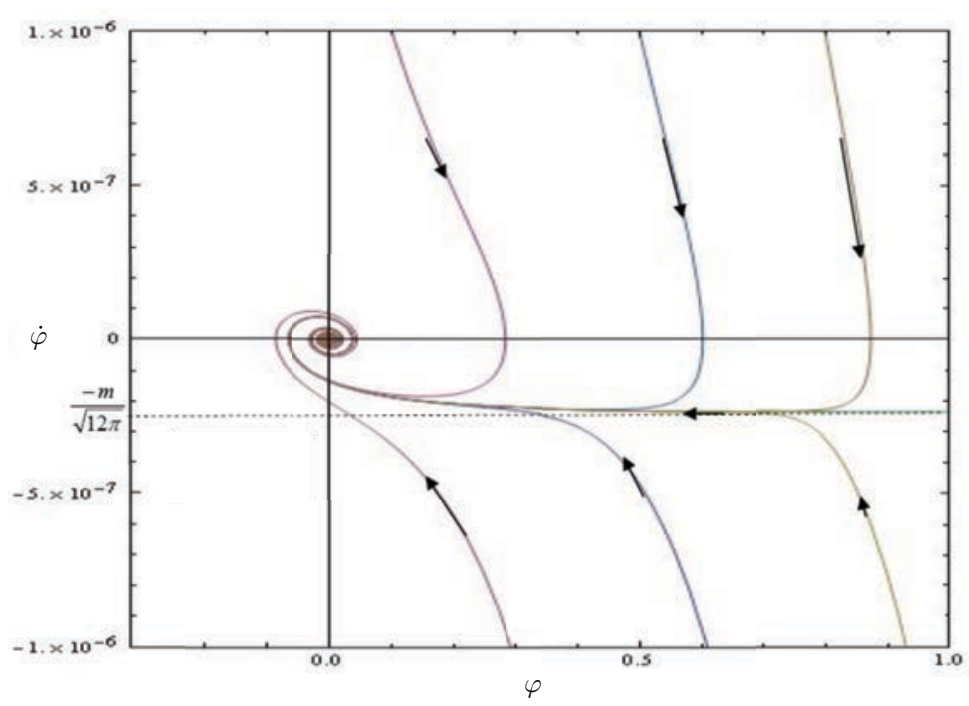

Fig. 2. The phase diagram for the chaotic inflation. The dashed line indicates the attractor solution to the inflaton $\varphi$, which corresponds to the slow roll regime of inflation.
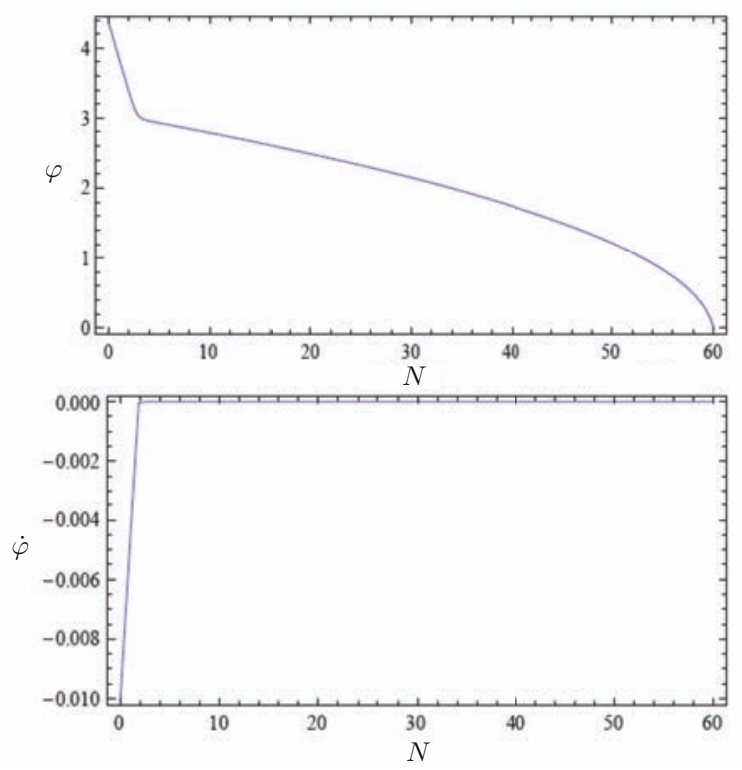

Fig. 3. Changes in $\varphi$ and $\dot{\varphi}$ against the inflationary e-folds. As the inflaton being placed initially at a position high enough, the magnitude of its velocity $|\dot{\varphi}|$ will decrease rapidly before the inflaton entering the slow roll regime. 


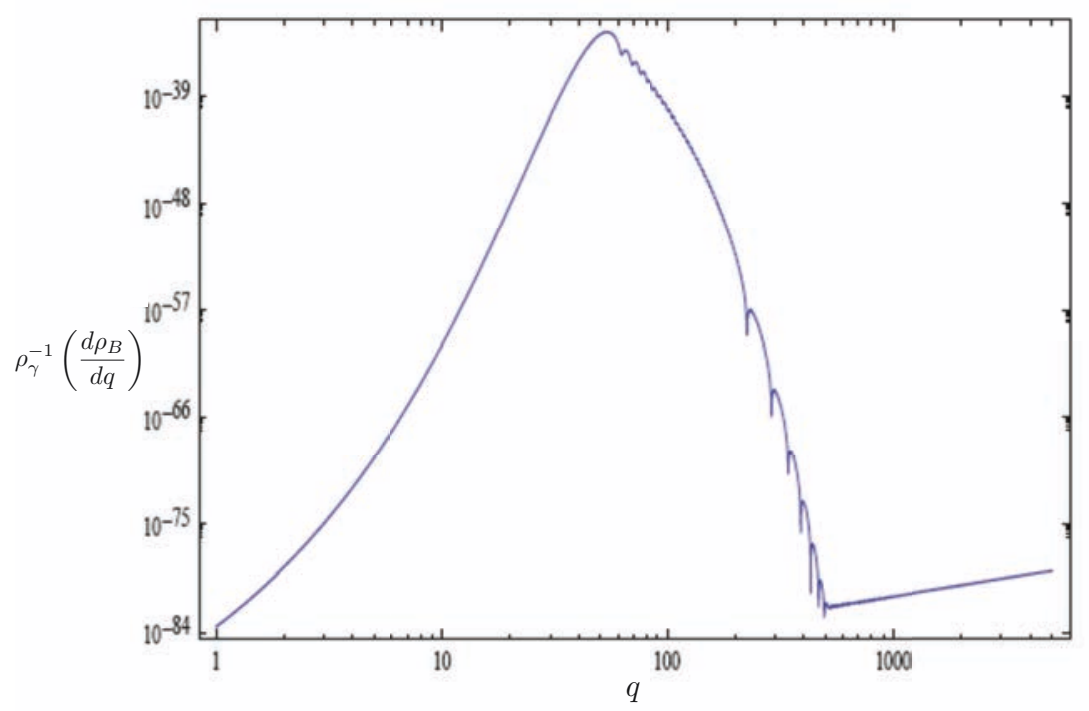

Fig. 4. The ratio of the spectral magnetic energy density to the energy density of photon at the end of inflation era. The coupling $c=34.5$ in this diagram. The peak mode with $q \simeq 53$ corresponds to a comoving distance of about $100 \mathrm{Mpc}$.

\section{Acknowledgments}

This work was supported in part by the National Science Council, Taiwan, ROC under the Grants NSC 97-2112-M003-004-MY3 (W.-L. Lee) and NSC 98-2112-M001-009-MY3 (K.-W. Ng); by the Focus Group of Cosmology and Particle Astrophysics (FGCPA) of National Center of Theoretical Sciences (NCTS), Taiwan; and by Leung Center for Cosmology and Particle Astrophysics (LeCosPA), National Taiwan University.

\section{References}

1. P. P. Kronberg, Rep. Prog. Phys. 57 (1994) 325.

2. L. M. Widrow, Rev. Mod. Phys. 74 (2002) 775.

3. E. N. Parker, Astrophys. J. 163 (1971) 255; Cosmical Magnetic Fields (Clarendon, Oxford, England, 1979); Ya. B. Zel'dovich, A. A. Ruzmaikin, and D. D. Sokoloff, Magnetic Fields in Astrophysics (Gordon and Breach, New York, 1983).

4. L. Biermann, Z. Naturf. 5A (1950) 65.

5. E. Harrison, Phys. Rev. Lett. 18 (1967) 1011; Phys. Rev. 167 (1968) 1170; Mon. Not. R. Astr. Soc. 147 (1970) 279.

6. M. Giovannini, Magnetic fields, strings and cosmology, in String theory and fundamental interactions, eds. M. Gasperini and J. Maharana (Springer Berlin/Heidelberg, 2007).

7. For a review, see K. Subramanian, Astro. Nachr. 331 (2010) 110. 
8. M. S. Turner and L. M. Widrow, Phys. Rev. D 37 (1988) 2743.

9. B. Ratra, Astrophys. J. 391 (1992) L1; M. Gasperini, M. Giovannini and G. Veneziano, Phys. Rev. Lett. 75 (1995) 3796; M. Giovannini, Phys. Rev. D 64 (2001) 061301; K. Bamba and J. Yokoyama, Phys. Rev. D 69 (2004) 043507; Phys. Rev. D 70 (2004) 083508; K. Bamba and M. Sasaki, JCAP 02 (2007) 030.

10. W. D. Garretson, G. Field, and S. Carroll, Phys. Rev. D 46 (1992) 5346; G. Field and S. Carroll, Phys. Rev. D 62 (2000) 103008.

11. M. Giovannini and M. Shaposhnikov, Phys. Rev. D 62 (2000)103512.

12. J. Martin and J. Yokoyama, JCAP 01 (2008) 025.

13. V. Demozzi, V. Mukhanov and H. Rubinstein, JCAP 08 (2009) 025.

14. R. Durrer, L. Hollenstein and R. K. Jain, arXiv: 1005.5322 (2010).

15. D.-S. Lee, W. Lee and K.-W. Ng, Phys. Lett. B 542 (2002) 1.

16. W. Lee and K.-W. Ng, Phys. Rev. D 67 (2003) 107302.

17. A. D. Linde, JETP Lett. 38 (1983) 176.

18. V. Mukhanov, Physical Foundations of Cosmology (Cambridge University Press, New York, 2005).

19. G. G. Raffelt, Stars as Laboratories for Fundamental Physics (University of Chicago Press, Chicago, 1996). 